$5-1-2012$

\title{
Steady State Probabilities of a Three Preemptive Single Server Queue
}

Ameen Jameel Alawneh

Qatar University

Follow this and additional works at: http://digitalcommons.wayne.edu/jmasm

Part of the Applied Statistics Commons, Social and Behavioral Sciences Commons, and the Statistical Theory Commons

\section{Recommended Citation}

Alawneh, Ameen Jameel (2012) "Steady State Probabilities of a Three Preemptive Single Server Queue," Journal of Modern Applied Statistical Methods: Vol. 11 : Iss. 1 , Article 17.

DOI: $10.22237 /$ jmasm/1335845760

Available at: http://digitalcommons.wayne.edu/jmasm/vol11/iss1/17

This Regular Article is brought to you for free and open access by the Open Access Journals at DigitalCommons@WayneState. It has been accepted for inclusion in Journal of Modern Applied Statistical Methods by an authorized editor of DigitalCommons@WayneState. 


\title{
Steady State Probabilities of a Three Preemptive Single Server Queue
}

\author{
Ameen Jameel Alawneh \\ Qatar University, \\ Qatar, Qatar
}

A three preemptive priority queuing system is considered where customers with three priorities joined a queue according to a Poisson process. A customer with higher priority needs to enter the service immediately upon arrival. The recursive formulas approach was extended to determine the steady state probabilities of such a priority queuing system.

Key words: Poisson process, recursive formulas based approach, steady state probabilities, three preemptive queues.

\begin{abstract}
Introduction
Queuing systems are used frequently and represent adequate models in many real life aspects. Examples of queuing systems include job processing, production lines, service centers (such as ATM machines), bus stations, phone calls and network service. Consider an $\mathrm{M} / \mathrm{M} / 1$ queuing system where customers arrive to a service facility with one server; the arrival process is Poisson with rate $\lambda$, and the service time distribution is exponential with rate $\mu$. If $\rho(=\lambda / \mu)<1$, then the system is in a steady state and steady state probabilities exist. Determining performance measures for $\mathrm{M} / \mathrm{M} / 1$ is simple and can be found in most elementary queuing system texts, such as Gross and Harries (1998).

Priority queues form a large class of queuing models where arrival customers are distinguished according to their importance. The customer with higher priority should be served before those of lower priorities. Examples include analysis of computer and communication systems, mobile phone
\end{abstract}

Ameen Alawneh is an Associate Professor of Statistics and Operations Research. $\mathrm{He}$ is on leave from JUST in Jordan and is currently working in the Department of Math, Statistics and Physics at Qatar University. His main research interest focuses on the stochastic process. Email him at: aalawneh@qu.edu.qa. networks, ambulances and surgeries. The performance analysis of multi-server queues with multiple classes of customers often experiences difficulty because the state space needed to capture the system behavior grows infinitely in multiple dimensions; Marks (1973) and Cidon and Sidi (1990) proposed different approaches to obtain the steady state probabilities of such systems.

\section{Two Levels Priority Queues}

Consider an $\mathrm{M} / \mathrm{M} / 1$ with two priority levels of customers: high and low. The high priority customers need to be served ahead of the low priority customers. The arrival process is Poisson with rates $\lambda_{1}$ and $\lambda_{2}$ for high and low priority customers, respectively. Also, the service time distribution is exponential with rates $\mu_{1}$ and $\mu_{2}$ for high and low priority customers, respectively. The service discipline within each priority level is first in, first out (FIFO).

Preemptive queues indicate that a customer with high priority must be served upon arriving unless there are already high priority customers in the queue or in service, that is., ahead of any low priority customers in the system. Thus, upon arrival of a high priority customer, if the customer in service is low priority then he/she is ejected from service and the high priority customer is serviced; the low priority customer returns to the service center and re-starts service from the beginning. Nonpreemptive queues are defined as those where a 
customer in service cannot be ejected from service upon the arrival of high priority customer.

Steady State Probabilities of Priority Queuing Systems

The steady state probabilities of queuing systems can be determined with ease when the queue is stable, however, it is not an easy task in the case of infinite queues, when the system has a very large number of states or when $\rho$ (the intensity factor) approaches 1 (Smith, 2002). Different approaches have been proposed in the literature to find steady state probabilities. Some are based on generating functions, difference equations or direct algebraic manipulations (Mark, 1973; Cidon \& Sidi, 1990). In their work, Smith (2002) and Osogami, et al. (2004) considered approaches based on manipulating queuing systems as Markov chains.

The limiting distribution of a Markov chain can be interpreted as a steady state probability. Another approach is termed indirect; this approach is based on identifying the stationary distribution of a Markov chain associated with the number of customers at a moment when a customer finishes service and leaves the system. The limiting distribution of the Markov chain is the steady state probability of the corresponding queuing system. Many authors have used this indirect approach in the literature (Osogami, et al.; 2004; Sheskin, 1985). Gail, et al. (1992) considered a Markov chain with two priorities and multiple servers: again, when the queue length is infinite, determining the stationary distribution of a Markov chain becomes challenging.

Heyman (1990) proposed an approach to ascertain approximate values of a stationary distribution of an infinite stochastic matrix in one dimension. Heyman's approach was extended to the case of two dimensional state space Markov chains and applied to a nonpreemptive queuing system (Alawneh, 1995). The truncated approach was used by Alawneh (2011) to determine approximate values of the steady state probabilities of $\mathrm{M} / \mathrm{M} / 2$ with infinite queues; results were compared with approaches put forth by Flatto and McKean (1977) and Flatto and Han (1977). This article presents an extension of a recursive formula based approach that may be used to find the steady state probabilities of three preemptive priority levels queuing system with one server.

Steady State Probabilities of Two Preemptive Priority Queue

Marks (1973) was the first to study nonpreemptive queuing systems. He developed a computational approach based on recursion formulas to determine the exact values of both preemptive and non-preemptive systems. Cidon \& Sidi (1990) developed a recursive formula based on a moment generating function to find the same probabilities. However, as model complexity increases, the required algebraic manipulations become more tedious. Pasternack and Drezener (1998) proposed a recursive formula based on difference equations to establish exact probabilities for priority queues; their technique requires less computation than Mark's (1973). Smith (2002) and Pasternack and Drezener (1998) proposed an alternate approach for use when a system is finite but the expected number of customers is large.

A recursive formula approach introduced in the literature to determine the exact values of the steady state probabilities of M/M/1 for customers with two priorities - high and low - was provided by Marks (1973). Consider an M/M/1 where arrival customers are classified into two types according to their priority of obtaining service: high and low. Four possible cases are possible for the state space: both $m=0$ and $n=0$, only $m=0$, only $n=0$; or $(n, m) \neq(0,0)$. Figure 1 illustrates the general case when both $\mathrm{m}$ and $\mathrm{n}$ are nonzero; that is, $(n$, $m) \neq(0,0)$. The other three cases may be obtained by using an appropriate substitution of $n$ and/or $m$.

Let $\mathrm{P}_{n m}$ be the probability of having $n$ high and $m$ low priority customers in the system at the moment when one customer finishes his service and leaves the system, where $n$ and $m$ are non-negative integers. The steady state probability is determined by solving equations (White \& Christie, 1958):

$$
\mu_{1} P(1,0)-\mu_{2} P(0,1)=\lambda P(0,0)
$$


Figure 1: Special Case of a State Diagram of M/M/1 with Two Priorities

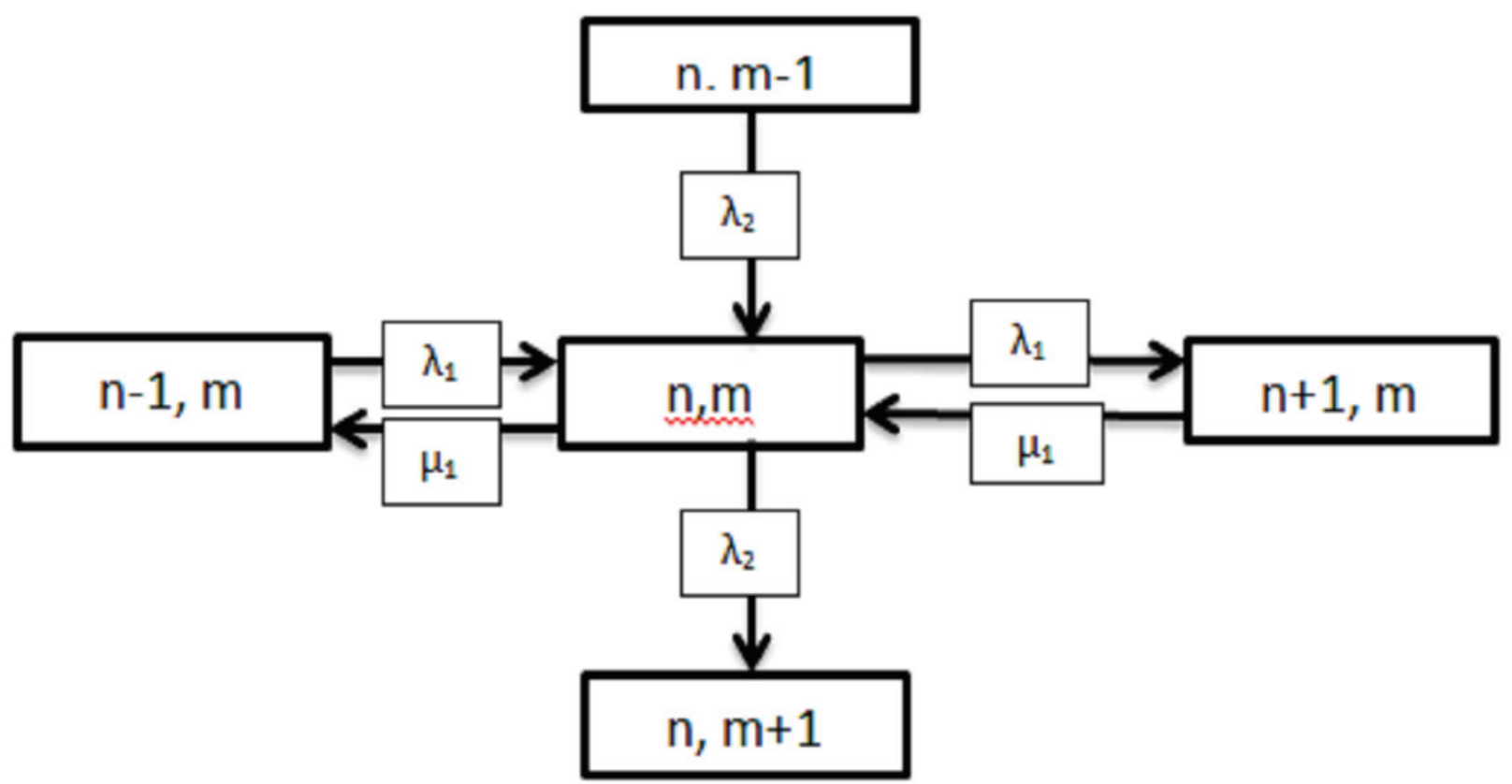

$\mu_{1} P(n+1,0)-\lambda_{1} P(n-1,0)=\left(\lambda+\mu_{1}\right) P(n, 0)$

$$
\left[\begin{array}{c}
\mu_{1} P(1, m)+\mu_{1} P(1, m) \\
+\mu_{2} P(1, m+1)+\lambda_{2} P(0, m-1)
\end{array}\right]=\left(\lambda+\mu_{2}\right) P(0, m)
$$

$$
\left[\begin{array}{c}
\mu_{1} P(n+1, m)+\lambda_{2} P(n, m-1) \mu \\
+\lambda_{2} P(n-1, m)
\end{array}\right]=\left(\lambda+\mu_{1}\right) P(n, m)
$$

for $n, m=0,1,2, \ldots$

The following ten-step algorithm was developed by Marks (1973) and may be used to solve equations (1) to (4) in order to determine the steady state probabilities of two preemptive queues:

1. Calculate $P_{00}=1-\rho_{1}-\rho_{2}$.

2. Set $\mathrm{B}_{00}=\mathrm{p}_{00}$.

3. Set $m=1$.
4. Calculate $\mathrm{B}_{11}$ as:

$$
B_{11}=\frac{1}{[\beta-\alpha]} \gamma \rho_{2} B_{00},
$$

where $0<\gamma<\infty$.

5. Calculate $\mathrm{B}_{01}$ as:

$$
B_{01}=\frac{[\beta-1]}{\gamma} B_{00}
$$

where

$$
\begin{aligned}
& \beta= \\
& \frac{1}{2}\left[\left(\rho_{1}+\gamma \rho_{2}+1\right)+\sqrt{\left(\rho_{1}+\gamma \rho_{2-}+1\right)^{2}-4 \rho_{1}}\right] .
\end{aligned}
$$

6. Increase $m$ by one.

7. Calculate $B_{i m}$ for $\mathrm{i}=\mathrm{m}, \mathrm{m}-1, \ldots, 1$, using the equation: 


$$
\begin{aligned}
& B_{i m}= \\
& {\left[\frac{1}{\beta-\alpha} i\right]\left\{\gamma \rho^{2} B_{i-1, m-1}+\sum_{k=i+1}^{k=m}\left(\begin{array}{c}
K \\
i-1
\end{array}\right)\left[\alpha+\beta(-1)^{k-i+1}\right] B_{k m l}\right\}}
\end{aligned}
$$

for $\mathrm{i}=m, m-1, \ldots, 1 ; m \geqq 1$.

8. Calculate $\mathrm{B}_{0 m}$ using:

$$
\begin{aligned}
& B_{0 m}= \\
& \left(\frac{\omega}{\gamma}\right) B_{0, m-1}-\rho_{2} B_{0, m-2}-\left(\frac{\alpha}{\gamma}\right) \sum_{k=0}^{m-1} B_{k, m-1}
\end{aligned}
$$

and

$$
\begin{aligned}
& \beta, \alpha= \\
& \frac{1}{2}\left[\left(\rho_{1}+\gamma \rho_{2}+1\right) \pm \sqrt{\left(\rho_{1}+\gamma \rho_{2}+1\right)^{2}-4 \rho_{1}}\right],
\end{aligned}
$$

where $0<\alpha<1, \beta>1, \omega=\rho_{1}+\rho_{2}+\gamma$.

9. Repeat steps 6 through 8 until all necessary coefficients are obtained.

10. Calculate the state probabilities $\mathrm{P}_{n m}$ using the formula:

$$
\begin{gathered}
P_{n m}=\alpha^{n} \sum_{k=0}^{k=m} B_{k m} \eta^{k}, \\
n \geq 0, m \geq 0 .
\end{gathered}
$$

Steady State Probabilities of Three Preemptive M/M/1 Queue

Next, the recursive formula approach is extended to the case where there are three preemptive priority levels. In this case, customers are classified according to their priorities into three types or classes. Type I, the highest priority, is followed by Type II, and lastly Type III, which is without priority. Assuming a preemptive priority, meaning customers with higher priority enter service upon arrival and ahead of any customer from a lower priority. In addition, a customer from the higher priority may eject any lower priority customer in service. The state space of the queuing system depends on the number of customers from each priority level in the system - both in the queue and in service. If $n, m$ and $l$ are the number of each priority level in the system, then all $n, m$ and $l$ are nonnegative integers. Eight possible cases for the state space may be considered based on $n, m$ and $l$. Figure 2 illustrates the most general case when $n, m$ and $l$ are all positive; the other seven cases may be obtained by appropriate substitutions of the values of $n, m$ and $l$.

If $\lambda_{i}$ for $i=1,2,3$ is the arrival rate from each priority level and $\mu_{i}$ is the service rate for each priority level, for $i=1,2,3$, then

$$
\rho_{i}=\frac{\lambda_{i}}{\mu_{i}} \text { for } i=1,2,3 \text {, }
$$

and $\lambda$ and $\mu$ are defined as: $\lambda=\lambda_{1}+\lambda_{2}+\lambda_{3}$ and $\mu=\mu_{1}+\mu_{2}+\mu_{3}$, respectively.

Steady state equations are derived from Figure 2 as follows:

$$
\mu_{1} P_{0,1,0}+\mu_{2} P_{0,1,0}+\mu_{3} P_{0,0,1}=\lambda P_{0,0,0}
$$

$$
\mu_{1} P_{n+1,0,0}+\lambda_{1} P_{n-1,0,0}=\left(\lambda+\mu_{1}\right) P_{n, 0,0}
$$

$$
\begin{gathered}
\mu_{1} P_{1, n, 0}+\mu_{2} P_{0, n+1,0}+\lambda_{2} P_{0, n-1,0}= \\
\left(\lambda+\mu_{3}\right) P_{0, n}
\end{gathered}
$$

$$
\begin{gathered}
\mu_{1} P_{1,0, l}+\mu_{2} P_{0,1, l}+\mu_{3} P_{0,0, l+1}+\lambda_{3} P_{0,0, l-1}= \\
\left(\lambda+\mu_{3}\right) P_{0,0, l} \\
\mu_{1} P_{n, m, 0}+\lambda_{1} P_{n-1, m, 0}+\lambda_{2} P_{n-1, m, 0}= \\
\left(\lambda+\mu_{1}\right) P_{n, m, 0} \\
\mu_{1} P_{n+1,0, l}+\lambda_{1} P_{n-1,0, l}+\lambda_{3} P_{n-1,0, l-1}= \\
\left(\lambda+\mu_{1}\right) P_{n, 0, l}
\end{gathered}
$$




\section{AMEEN JAMEEL ALAWNEH}

Figure 2: Special Case of the State Diagram of $M / M / 1$ with Three Priorities

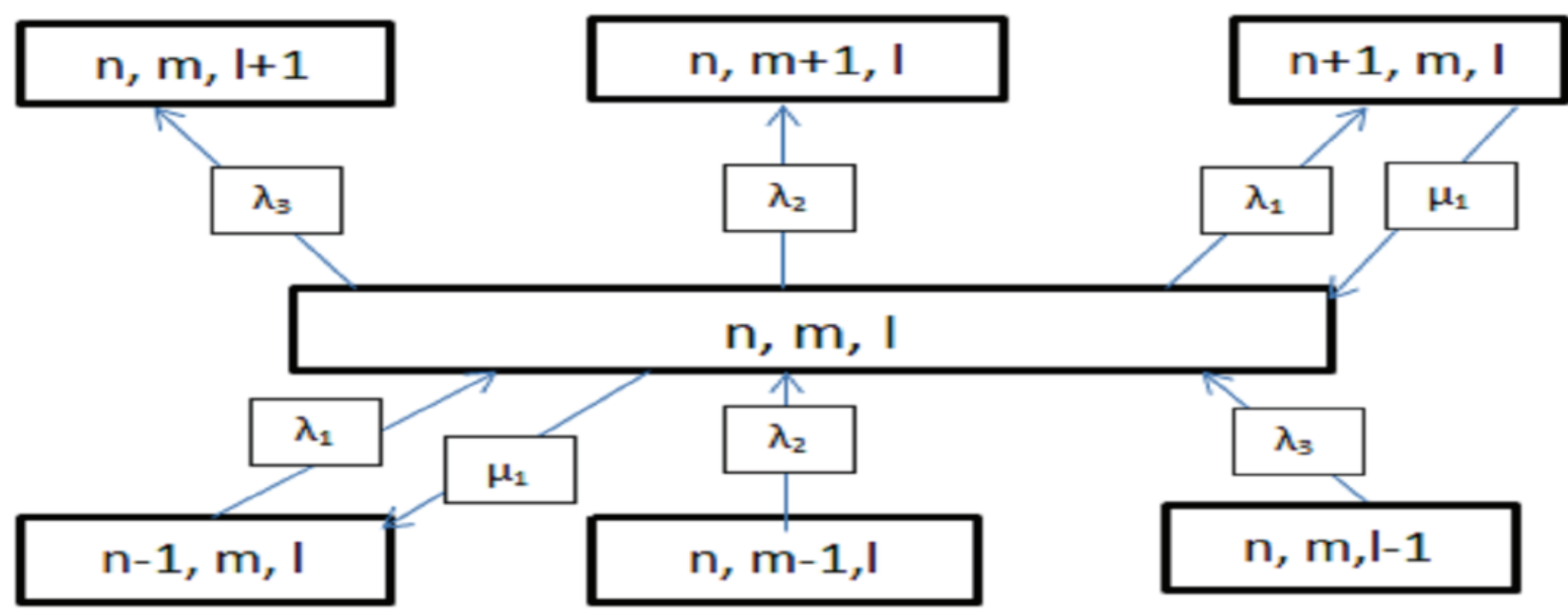

$$
\begin{gathered}
\mu_{1} P_{1, m, l}+\lambda_{2} P_{0, m-1, l}+\mu_{2} P_{0, m+1, l}+\lambda_{3} P_{0, m, l-1}= \\
\left(\lambda+\mu_{3}\right) P_{0, m, l}
\end{gathered}
$$

$$
\begin{gathered}
\mu_{1} P_{n+1, m, l}+\lambda_{1} P_{n-1, m, l}+\lambda_{2} P_{n, m-1, l}+\lambda_{3} P_{n, m, l-1}= \\
\left(\lambda+\mu_{1}\right) P_{n, m, l}
\end{gathered}
$$

and

$$
\sum_{l=0}^{\infty} \sum_{m=0}^{\infty} \sum_{n=0}^{\infty} P_{l, m, n}=1
$$

Defining $E P_{n, m, l}=P_{n+1, m, l}$ where $E$ is a difference operator and rewriting (2) using the difference operator $E$ results in:

$$
\begin{aligned}
\left(\mu_{1} E^{2}-\left(\lambda+\mu_{1}\right) E+\lambda_{1}\right) P_{l, 0,0} & =0 \\
& =\Psi(E) P_{l 00}
\end{aligned}
$$

where

$$
\Psi(E)=\left(\mu_{1} E^{2}-\left(\lambda+\mu_{1}\right) E+\lambda_{1}\right) .
$$

Substituting $m=1$ and rewriting (18) using difference operator $E$ results in:

$$
\begin{gathered}
\mu_{1} P_{n+1,1, l}+\lambda_{1} P_{n-1,1, l}+\lambda_{2} P_{n, 0, l}+\lambda_{3} P_{n, 0, l-1}= \\
\left(\lambda+\mu_{1}\right) P_{n, 0, l}
\end{gathered}
$$

and

$$
\Psi(E) P_{n, 1, m}=-\lambda_{3} P_{n, 0, l-1}-\lambda_{2} P_{n, 0, l}
$$

Using mathematical induction, for $n=0$,

$$
\Psi(E) P_{n, 1,0}=-\lambda_{2} P_{n, 0,0}
$$

and for $n=1$,

$$
\Psi(E) P_{n, 1,1}=-\lambda_{3} P_{n, 0,0}-\lambda_{2} P_{n, 0,1},
$$

$$
P_{n, 1,0}=B^{100} r^{n}+B_{101} n r^{n}
$$

and

$$
P_{n, 1,0}=B_{110} r^{n}+B_{111} n r^{n}+B_{112} n^{2} r n .
$$


Thus the general solution is

$$
P_{n, 1, l}=r^{n} \sum_{j=0}^{n+1} B_{i, m, j} n^{j}
$$

and, the general solution for the steady state probabilities is

$$
P_{n, m, l}=r^{n} \sum_{j=0}^{n+m} B_{i, m, j} n^{j}
$$

which shows that a recursive formula approach may be used to determine the steady state probabilities of a three preemptive queue.

Expected Number of Customers and the Average Waiting Time in $\mathrm{M} / \mathrm{M} / 1$

To compare an $\mathrm{M} / \mathrm{M} / 1$ with three priority levels but without priority according to the expected number of customers and average waiting time in the queue, let $\mathrm{L}_{i q}, i=1,2,3$ be the expected number of customers (average queue length) from the $i^{\text {th }}$ priority level in the queue, and let $\mathrm{W}_{i q}$ be the average waiting time for the $i^{\text {th }}$ priority level. The number of expected customers in the queue from the $i^{\text {th }}$ priority level is found using:

$$
\begin{aligned}
& L_{i q}=\frac{\rho_{i} \sum_{n=1}^{i} \rho_{n}}{\left(1-\sum_{n=1}^{i-1} \rho_{-} n\right)\left(1-\sum_{n=1}^{i} \rho_{n}\right)}, \\
& i=1,2,3 \text { and } \sum_{j=1}^{i} \rho_{j}<1 .
\end{aligned}
$$

If $\mathrm{L}_{q}$ is the expected number of customers in the queue then:

$$
L_{q}=\frac{1}{\lambda}\left(\lambda_{1} L_{1 q}+\lambda_{2} L_{2 q}+\lambda_{3} L_{3 q}\right)
$$

and the average waiting times in the queue is

$$
W q=\frac{1}{\lambda}\left(\begin{array}{lll}
\lambda_{1} & W_{1 q}+\lambda_{2} & W_{2 q}+\lambda_{3} W_{3 q}
\end{array}\right)
$$

where the average waiting time for the $i^{\text {th }}$ priority level is

$$
W_{i q}=\frac{L_{i q}}{\lambda}
$$

for $i=1,2,3$.

The expected number of customers and the average waiting times for an $\mathrm{M} / \mathrm{M} / 1$ without priorities are:

$$
\begin{gathered}
L_{q}=\frac{\rho^{2}}{1-\rho} \\
\text { and } \\
W_{q}=\frac{L_{q}}{\lambda}
\end{gathered}
$$

where the arrival rate for the non-priority is $\lambda=$ $\lambda_{1}+\lambda_{2}+\lambda_{3}$, and where $\lambda_{1}, \lambda_{2}$ and $\lambda_{3}$ are the same as in the priority queue. In addition, $\mu=\mu_{1}$ $+\mu_{2}+\mu_{3}$ and $\mu_{1}, \mu_{2}$ and $\mu_{3}$ are also the same as in the priority queue.

For the purpose of numerical comparisons between priority and non-priority customers, Table 1 shows different values for arrival and service rates for a priority model assuming $\lambda=\lambda_{1}+\lambda_{2}+\lambda_{3}$, and $\mu=\mu_{1}+\mu_{2}+\mu_{3}$ when customers are not prioritized.

\section{Conclusion}

Based on results shown in Table 1, the following conclusions are put forth:

1. The queue length in the non-priority systems always constitutes an upper bound for the queue length in the priority system.

2. The average waiting time for the nonpriority customers is an upper bound for the priority system.

3. Priority queues are more efficient when customers are classified according to their importance or needs for service.

4. As the intensity factor approaches one, the expected number of customers and the average waiting times increase for the highest priority customers.

This study shows that the recursive formal based approach may be used to find exact values of steady state probabilities for a three priority 
queuing system. Priority queues are more efficient than non-priority, particularly when customer arrivals are classified according to their importance or their service needs. In real life applications the number of the highest priority customers is limited; therefore, imposing such a condition on queue length will make using the recursive technique much easier.

Table 1: Expected Queue length and Waiting times for different values of $\mu$ and $\lambda$

\begin{tabular}{|c|c|c|c|c|c|c|c|c|}
\hline \multirow{2}{*}{$\mu$} & \multirow{2}{*}{$\lambda$} & \multirow{2}{*}{$\rho_{1}$} & \multirow{2}{*}{$\rho_{2}$} & \multirow{2}{*}{$\rho_{3}$} & \multicolumn{2}{|c|}{$\mathrm{L}_{\mathrm{q}}$} & \multicolumn{2}{|c|}{$\mathrm{W}_{\mathrm{q}}$} \\
\hline & & & & & With & Without & With & Without \\
\hline 6 & 3 & 0.167 & 0.167 & 0.167 & 0.128 & 0.500 & 0.064 & 0.167 \\
\hline 6 & 4 & 0.167 & 0.333 & 0.167 & 0.375 & 1.333 & 0.156 & 0.167 \\
\hline 6 & 4 & 0.167 & 0.167 & 0.333 & 0.533 & 1.333 & 0.267 & 0.167 \\
\hline 6 & 5 & 0.167 & 0.333 & 0.333 & 1.500 & 5.143 & 0.625 & 1.029 \\
\hline 6 & 4 & 0.167 & 0.333 & 0.167 & 0.375 & 1.333 & 0.156 & 0.167 \\
\hline 6 & 5 & 0.167 & 0.167 & 0.333 & 1.500 & 5.143 & 0.625 & 0.570 \\
\hline 7 & 5 & 0.167 & 0.333 & 0.333 & 0.918 & 0.714 & 0.394 & 0.357 \\
\hline 7 & 6 & 0.143 & 0.143 & 0.429 & 3.215 & 5.143 & 1.378 & 0.857 \\
\hline 7 & 6 & 0.143 & 0.143 & 0.571 & 1.615 & 5.143 & 0.519 & 0.857 \\
\hline 7 & 4 & 0.143 & 0.571 & 0.143 & 0.178 & 1.752 & 0.051 & 0.357 \\
\hline 7 & 5 & 0.286 & 0.143 & 0.143 & 0.426 & 1.786 & 0.106 & 0.357 \\
\hline 7 & 5 & 0.286 & 0.286 & 0.143 & 0.576 & 1.786 & 0.165 & 0.357 \\
\hline 7 & 4 & 0.286 & 0.143 & 0.286 & 0.178 & 1.752 & 0.051 & 0.355 \\
\hline 7 & 5 & 0.286 & 0.143 & 0.143 & 0.426 & 0.714 & 0.106 & 0.357 \\
\hline 7 & 6 & 0.286 & 0.286 & 0.286 & 0.576 & 5.143 & 0.165 & 0.857 \\
\hline 7 & 6 & 0.286 & 0.143 & 0.286 & 1.549 & 5.143 & 0.387 & 0.857 \\
\hline 7 & 6 & 0.286 & 0.286 & 0.429 & 2.313 & 5.143 & 0.661 & 0.857 \\
\hline 7 & 5 & 0.286 & 0.143 & 0.143 & 0.426 & 0.714 & 0.102 & 0.357 \\
\hline 7 & 6 & 0.429 & 0.143 & 0.143 & 1.077 & 5.143 & 0.231 & 0.857 \\
\hline 7 & 6 & 0.429 & 0.286 & 0.286 & 1.550 & 5.143 & 0.369 & 0.857 \\
\hline 7 & 6 & 0.571 & 0.143 & 0.143 & 1.147 & 5.143 & 0.246 & 0.857 \\
\hline
\end{tabular}




\section{References}

Alawneh, A. (1995). Approximating the stationary distribution of two-dimensional Markov chains. Unpublished Ph.D. Thesis, New Mexico State University, USA.

Alawneh, A. (2011). Numerical approach to compute the steady state probabilities of $\mathrm{M} / \mathrm{M} / 2$ with infinite queues. Accepted. International Journal of Applied Mathematics.

Ching, W., Zhang, S., Ng, M., \& Akustu, T. (2007). An approximation method for solving the steady state probability distribution of probabilistic Boolean networks. Bioinformatics, 23(12), 1611-1518.

Cidon, I., \& Sidi, M. (1990). Recursive computation of steady state probabilities in priority queues. Operations Research Letters, 9 , 249-256.

Flatto, L., \& McKean, H. P. (1977). Two queues in parallel. Communications on pure and applied mathematics, 30(2), 255-263.

Gail, H. R., Hantler, S. L., \& Taylor, B. A. (1992). On a preemptive Markovian queue with multiple servers and two priority classes. Mathematics of Operations Research, 17(2), 365-391.

Gross, D., \& Harris, C. (1998). Fundamentals of queuing systems, $3^{\text {rd }}$ Edition. New York, NY: John Wiley.

Heyman, D. P. (1991). Approximating the stationary distribution of an infinite stochastic matrix. Journal of Applied Probability, 28(1), 96-103.
Mark, P., \& Karlin, S. (2011). An Introduction to stochastic modeling, $4^{\text {th }}$ Edition. London: Academic Press.

Marks, B. I. (1973). State probabilities of $\mathrm{M} / \mathrm{M} / 1$ priority queues. Operations Research, 21, 974-987.

Miller, D. G. (1981). Computation of steady state probabilities for $\mathrm{M} / \mathrm{M} / 1$ priority queues. Operations Research, 29, 945-958.

Osogami, T., Wierman, A., HarcholBalter, M., \& Scheller-Wolf, A. (2004). A recursive analysis technique for multidimensionally infinite Markov chains. $A C M$ SIGMETRICS Performance Evaluation Review, 32(2), 3-5.

Pasternack, B. A., \& Drezener, Z. (1998). A note on calculating steady state results for $\mathrm{M} / \mathrm{M} / \mathrm{k}$ queuing systems when the ration of the arrival rate to the service rate is large. Journal of Applied Mathematics and Decision Science, 2(2), 201-203.

Sheskin, T. (1985). A Markov chain portioning algorithm for computing steady state probabilities. Operations Research, 33(1), 228235.

Smith, D. (2002). Calculation of M/M/ queues: Further approach. Journal of Applied Mathematics and Decision Science, 6(1), 43-50.

Wright, P. (1992). Two parallel processors with coupled inputs. Advances in Applied Probability, 24(4), 986-1007. 